EPJ Web of Conferences 69, 00018 (2014)

DOI: $10.1051 /$ epjconf/20146900018

(C) Owned by the authors, published by EDP Sciences, 2014

\title{
Photon strength functions in Gd isotopes studied from radiative capture of resonance neutrons
}

J. Kroll ${ }^{1, a}$, B. Baramsai², G. E. Mitchell ${ }^{2}$, U. Agvaanluvsan³ ${ }^{3}$, F. Bečvár ${ }^{1}$, T. A. Bredeweg ${ }^{4}$, A. Chyzh ${ }^{2}$, A. Couture ${ }^{4}$, D. Dashdorj ${ }^{3}$, R. C. Haight ${ }^{4}$, M. Jandel ${ }^{4}$, A. L. Keksis ${ }^{4}$, M. Krtička ${ }^{1}$, J. M. O'Donnell ${ }^{4}$, W. Parker ${ }^{5}$, R. S. Rundberg ${ }^{4}$, J. L. Ullmann ${ }^{4}$, S. Valenta ${ }^{1}$, D. J. Vieira ${ }^{4}$, C. Walker ${ }^{2}$, and C. Y. Wu ${ }^{5}$

${ }^{1}$ Charles University in Prague, CZ-180 00 Prague 8, Czech Republic

${ }^{2}$ North Carolina State University, Raleigh, North Carolina 27695, USA and Triangle Universities Nuclear Laboratory, Durham, North Carolina 27708, USA

${ }^{3}$ MonAme Scientific Research Center, P.O.Box 24-603, Ulaanbaatar, Mongolia

${ }^{4}$ Los Alamos National Laboratory, P.O. Box 1663, Los Alamos, New Mexico 87545, USA

${ }^{5}$ Lawrence Livermore National Laboratory, Livermore, California 94551, USA

\begin{abstract}
The experimental spectra of $\gamma$ rays following radiative neutron capture on isolated resonances of stable ${ }^{152,154-158} \mathrm{Gd}$ targets were measured by the DANCE calorimeter installed at the Los Alamos Neutron Scattering Center in New Mexico, USA. These spectra were analyzed within the extreme statistical model to get new information on the photon strength functions. Special emphasis was put on study of the scissors vibrational mode present in these isotopes. Our data show that the scissors-mode resonances are built not only on the ground states but also on the excited levels of all studied Gd isotopes. The scissors mode strength observed in ${ }^{157,159} \mathrm{Gd}$ products is significantly higher than in neighboring even-even nuclei ${ }^{156,158} \mathrm{Gd}$. Such a difference indicates the existence of an odd-even effect in the scissors mode strength. Moreover, there exists no universal parameter-free model of the electric dipole photon strength function describing the experimental data in all of the Gd isotopes studied. The results for the scissors mode are compared with the $\left(\gamma, \gamma^{\prime}\right)$ data for the ground-state transitions and with the results from ${ }^{3} \mathrm{He}$-induced reactions.
\end{abstract}

\section{Introduction}

In medium and heavy mass nuclei detailed information on the properties of nuclear levels and transitions between them exists usually only at low excitation energies above the ground state. In this region, the level spacing is sufficiently high to clearly observe individual transitions. As the nuclear level density (NLD) increases with excitation energy, it is almost impossible to resolve transitions to or from the individual levels. Obtaining reliable spectroscopic information on these levels, which form the so-called quasicontinuum, becomes very difficult. It is believed that properties of the nucleus in the quasicontinuum can be described by the extreme statistical model in terms of the NLD and a

\footnotetext{
ae-mail: kroll@ipnp.troja.mff.cuni.cz
} 
set of photon strength functions (PSFs) for different types and multipolarities of transitions. These statistical quantities are very important for the correct description of various reaction rates that are especially needed in nuclear astrophysics and in the development of advanced nuclear reactors. One of the ways to examine PSFs and the NLD at excitation energies up to the neutron separation energy $S_{\mathrm{n}}$ is via study of the $\gamma$ rays following the radiative neutron capture at isolated neutron resonances. Results of the analysis of the $\gamma$-ray spectra measured for ${ }^{152,154-158} \mathrm{Gd}$ targets at isolated $s$-wave neutron resonance are presented below.

\section{DANCE Experiment}

The measurement was performed using the highly segmented Detector for Advanced Neutron Capture Experiments (DANCE) detector array $[1,2]$ installed at the pulsed neutron beam at the Los Alamos Neutron Science Center (LANSCE) at Los Alamos National Laboratory [3] which produces a white spectrum of neutrons with energies from subthermal up to several $\mathrm{MeV}$ with the repetition rate of $20 \mathrm{~Hz}$. These neutrons are sent to flight path 14 at the Manuel Lujan Jr. Neutron Scattering Center.

The DANCE detector array $[1,2]$ is installed at $20.2 \mathrm{~m}$ on this flight path and consists of $160 \mathrm{BaF}_{2}$ scintillation crystals surrounding a sample and covering a solid angle of $\simeq 3.5 \pi$. A ${ }^{6} \mathrm{LiH}$ shell about $6-\mathrm{cm}$ thick is placed between the sample and the $\mathrm{BaF}_{2}$ crystals in order to reduce the background from capture of scattered neutrons on Ba isotopes in the crystals. The DANCE acquisition system [4] is based on waveform digitization of signals from all 160 detectors using Acqiris DC265 digitizers with a sampling rate of 500 megasamples per second.

The energy calibration of the DANCE crystals was performed with a combination of natural $\gamma$-ray sources at low $\gamma$-ray energies and the intrinsic $\alpha$-radioactivity of ${ }^{226} \mathrm{Ra}$ and its daughters present in the $\mathrm{BaF}_{2}$ crystals. The latter calibration was conducted on a run-by-run basis to provide the energy alignment of all crystals in the off-line analysis. The identification of $\alpha$ particles is possible from the ratio of the fast and slow components of the $\mathrm{BaF}_{2}$ signal [2].

Measurements were performed using isotopically enriched $\mathrm{Gd}$ targets. The ${ }^{155-158} \mathrm{Gd}$ targets were prepared at Oak Ridge National Laboratory as self-supporting metal foils. The ${ }^{152} \mathrm{Gd}$ and ${ }^{154} \mathrm{Gd}$ targets were prepared at Lawrence Livermore National Laboratory by the electroplating of enriched Gd on a Be foil which was glued to an aluminium ring. The isotopic composition of the targets together with their average thicknesses is listed in Ref. [5, 6].

\section{Data Reduction}

Only events from well-resolved resonances, that can be identified using time-of-flight (TOF) technique, were considered. Examples of TOF spectra for ${ }^{154} \mathrm{Gd}$ and ${ }^{158} \mathrm{Gd}$ targets are given in Fig. 1. Only $s$-wave neutron resonances were observed at low neutron energies in this mass region.

Usually a $\gamma$ cascade consists of several emitted $\gamma$ rays. The signals were assumed to originate from a single $\gamma$ cascade if they were detected within a 10 ns coincidence window. An emitted single $\gamma$ ray does not usually deposit its full energy in one crystal. Thus the number of crystals that fire is often higher than the physical multiplicity of the capture event. Therefore all contiguous crystals that have fired during a capture event are combined into clusters and considered as the response of the detector array to a single $\gamma$ ray. The number of clusters observed in a capture event is called the cluster multiplicity. The sum-energy spectra for different cluster multiplicities from several resonances of the ${ }^{158}$ Gd target are shown in Fig. 2(a).

Each sum-energy spectrum consists of (i) a peak near the full energy available from the neutron capture reaction - the full-energy peak - which is close to the neutron separation energy $S_{\mathrm{n}}$ for a given 

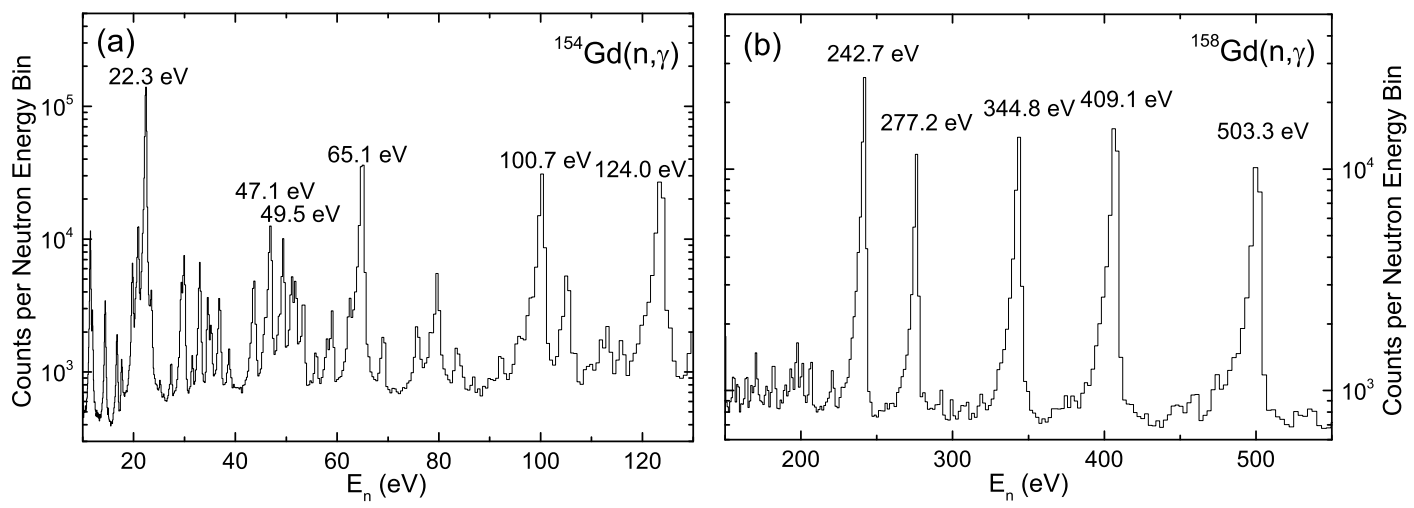

Figure 1. Time-of-flight spectra for ${ }^{154} \mathrm{Gd}$ (a) and ${ }^{158} \mathrm{Gd}$ (b) targets after transformation to a neutron-energy scale. There are 400 (exponentially distributed) neutron energy bins per decade. Only events corresponding to cluster multiplicities $M=2-7$ and a detected energy sum in the region of $5.8-6.6 \mathrm{MeV}$ (for ${ }^{154} \mathrm{Gd}$ ) and $5.2-6.2$ $\mathrm{MeV}$ (for ${ }^{158} \mathrm{Gd}$ ) were considered. Resonances used in the analysis of the MSC spectra are labeled. Many weak resonances, especially in the spectrum for ${ }^{154} \mathrm{Gd}$ target, come from ${ }^{155,157} \mathrm{Gd}$ impurities.
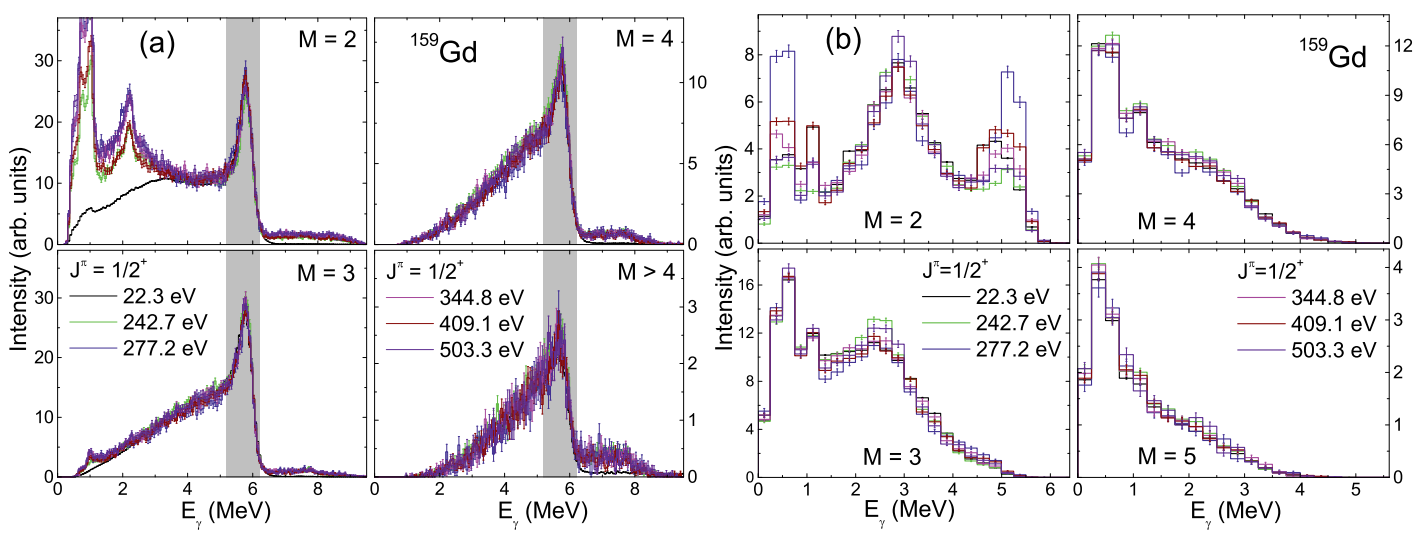

Figure 2. Experimental sum-energy (a) and MSC (b) spectra for cluster multiplicities $M=2-5$ for $J^{\pi}=1 / 2^{+}$ $s$-wave neutron resonances of the ${ }^{159} \mathrm{Gd}$ product nucleus. Energies of the neutron resonances are indicated. All resonances were normalized to the same number of captures that contribute to the gray areas. Only events from these gray areas were used for the construction of MSC spectra.

nucleus and (ii) a low-energy tail that corresponds to $\gamma$ cascades for which a part of the emitted energy escaped the detector array. The shape of the spectrum at low sum energies, approximately below $3 \mathrm{MeV}$, is strongly influenced by the background from natural $\beta$ activity in the $\mathrm{BaF}_{2}$ crystals, especially for low multiplicities. In practice, this background is not important for us as only events from the full-energy peak were used in our analysis. The events above $S_{\mathrm{n}}$ in the sum-energy spectra come from two sources. The dominant contribution, especially at low multiplicities, is from the capture of scattered neutrons in the barium of the $\mathrm{BaF}_{2}$ scintillation crystals, with neutron separation energies between $S_{\mathrm{n}}=4.723$ and $9.468 \mathrm{MeV}$ for ${ }^{139} \mathrm{Ba}$ and ${ }^{134} \mathrm{Ba}$, respectively. For higher multiplicities there 
is also a small contribution from the radiative neutron capture on ${ }^{155,157} \mathrm{Gd}$ sample impurities in the sum-energy spectra of even Gd targets.

The main subject of our analysis were so-called multistep cascade (MSC) spectra. These are constructed only from those events which fall into the full-energy peak in the sum-energy spectra. The ranges of the energy sums used in the construction of MSC spectra are illustrated by the grey areas in Fig. 2(a). The MSC spectrum for multiplicity $M$ is understood to be a spectrum of energies belonging to a single cascade that are deposited in $M$ detector clusters. Experimental MSC spectra constructed in this way contain a background. This background is usually very small for strong neutron resonances and was subtracted using MSC spectra from off-resonance regions. Experimental background-free MSC spectra from selected resonances of ${ }^{158} \mathrm{Gd}$ are shown in Fig. 2(b).

To facilitate the comparison of the experimental MSC spectra with the model predictions given below, all sum-energy and MSC spectra for a given resonance were normalized using one common factor. Specifically, we assume that the integral of the sum-energy spectra for multiplicities $M=2-7$ takes a value common for all resonances. As the spectrum for $M=1$ is strongly dominated by the background contributions, it is omitted from the analysis. Virtually no events for $M \geq 6$ or 7 were measured. For comparison with simulations the MSC spectra with $250 \mathrm{keV}$ wide bins were used. This width is comparable with the detector energy resolution. Use of these relatively wide energy bins allows reduction of the experimental uncertainties and simultaneously suppresses the fluctuations predicted by the simulations.

\section{Modelling of $\gamma$ decay}

MSC spectra are products of a complicated interplay between PSFs and the NLD. As a result, it is not straightforward to get information on these quantities from measured spectra. To learn about PSFs and the NLD we adopt a trial-and-error approach in which we compare the experimental MSC spectra with their simulated counterparts obtained from the generation of $\gamma$ cascades using the DICEBOX algorithm [7] for various models of the PSFs and the NLD followed by simulation of the detector response to these cascades using the GEANT4 code [8]. Such a comparison can tell us which models of PSFs and the NLD are most likely to be valid and which are incorrect. The main feature of the DICEBOX algorithm is that it takes into account all expected fluctuations involved in the $\gamma$ decay. Due to the presence of these fluctuations, which are dominated by the Porter-Thomas fluctuations of partial radiation widths [9], there exists an infinite number of so-called nuclear realizations (NRs) that differ from each other even for fixed models of PSFs and the NLD. About 20 independent NRs with given combination of PSFs and NLD were generated for each $J^{\pi}$ of the initial $s$-wave resonances with $10^{5}$ cascades in one NR. Generated cascades were then subject to a GEANT4-based simulation of the detector system response. The MSC spectra from simulations were constructed under the same condition on the detected energy sum as applied to the experimental spectra.

\subsection{Electric dipole transitions}

It is well known that for $\gamma$-ray energies above the neutron separation energy $S_{\mathrm{n}}$ the electric dipole (E1) transitions play a dominant role. The $E 1 \mathrm{PSF}$ at these energies in axially deformed nuclei seems to be fully consistent with the sum of the two Lorentzian terms of the giant dipole electric resonance (GDER). The model is known as the Standard Lorentzian (SLO) model. On the other hand, the shape of the $E 1$ PSF below $S_{\mathrm{n}}$ is not well known. There are many available parametrizations of the $E 1$ PSF in this region of $\gamma$-ray energies that modify the Lorentzian shape of the low-energy tail of the GDER.

Usually, either the model of Kadmenskij, Markushev, and Furman (KMF) [10] or the Enhanced Generalized Lorentzian (EGLO) [11] model is used at these energies. Many other models of the E1 

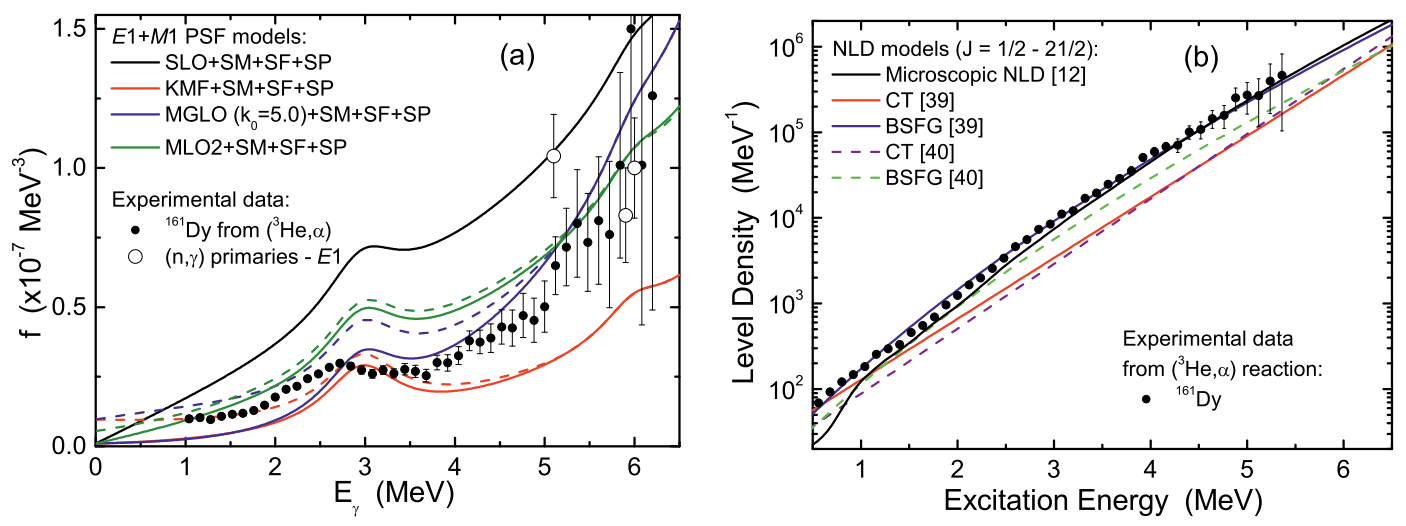

Figure 3. Left: Some of the $E 1+M 1$ composite PSF models used in our simulations for the ${ }^{159} \mathrm{Gd}$ product. Two curves for the KMF, MGLO and MLO2 models indicate how these models change as a function of nuclear temperature - the solid curve corresponds to $T_{\mathrm{f}}=0 \mathrm{MeV}$, while the dashed one to $T_{\mathrm{f}}=\sqrt{\left(S_{\mathrm{n}}-E_{\gamma}-\Delta\right) / a}$, where $a$ is the level density parameter and $\Delta$ is the pairing energy [39, 40, 42]. The parametrization of the GDER is taken from Ref. [43] for the ${ }^{160} \mathrm{Gd}$ nucleus. The $M 1$ PSF consists of a single-particle component $f_{M 1}^{(\mathrm{SP})}=1 \times 10^{-9} \mathrm{MeV}^{-3}$, a spin-flip part represented by the double-humped Lorentzian with the parametrization $E_{\mathrm{SF}, 1}=6 \mathrm{MeV}, \Gamma_{\mathrm{SF}, 1}=0.8 \mathrm{MeV}, \sigma_{\mathrm{SF}, 1}=0.7 \mathrm{mb}$ and $E_{\mathrm{SF}, 2}=8 \mathrm{MeV}$, GammasF, $=1.8 \mathrm{MeV}, \sigma_{\mathrm{SF}, 2}=1.1 \mathrm{mb}$, and the scissors mode $f_{M 1}^{(\mathrm{SM})}$ with the parametrization $E_{\mathrm{SM}}=3.0 \mathrm{MeV}, \Gamma_{\mathrm{SM}}=1.0 \mathrm{MeV}$ and $\sigma_{\mathrm{SM}}=0.7 \mathrm{mb}$. The experimental data for $f_{E 1}$ from the $(\mathrm{n}, \gamma)$ reaction are from the RIPL database $[44]\left({ }^{155,157} \mathrm{Gd}\right.$ at 5.9 and $6 \mathrm{MeV}$, respectively), and from Ref. [45] $\left({ }^{159} \mathrm{Gd}\right.$ at $\left.5.1 \mathrm{MeV}\right)$. Data from the $\left({ }^{3} \mathrm{He}, \alpha\right)$ reaction are from Ref. [16]. Right: The NLD models of ${ }^{159} \mathrm{Gd}$ summed over the spin range $J=1 / 2-21 / 2$ and both parities. The parameters of the CT and BSFG models are from [39] and [40]. The microscopic calculations are from RIPL-3 [12]. The experimental data are taken from the ${ }^{162} \mathrm{Dy}\left({ }^{3} \mathrm{He}, \alpha \gamma\right){ }^{161}$ Dy reaction measured by the Oslo group [16].

PSF, which have been tested in our analysis, can be found in the literature. We tested the following models which can be found in the RIPL-3 database [12]: the Hybrid model (GH) [13], the Generalized Fermi Liquid (GFL) model [14, 15], a family of three modified Lorentzian (MLO) models, and a PSF model originating from Hartree-Fock-Bogoliubov plus quasi-particle random-phase approximation (HFB-QRPA) microscopic calculations [12]. In addition to these models, we tested also the KMF model with constant nuclear temperature $T_{\mathrm{f}}(\mathrm{KMF}-\mathrm{T})$ which was often used in the analysis of data from ${ }^{3} \mathrm{He}$-induced reactions measured in the Oslo Cyclotron Laboratory [16-20] and the Modified Generalized Lorentzian (MGLO) model defined in Ref. [6] which is a modification of the EGLO model. There are two (ad-hoc) parameters, $k_{0}$ and $E_{\gamma 0}$, in the EGLO and MGLO models.

For a complete description of $\gamma$ decay one needs information on the PSFs at all excitation energies. In some models, the dependence on any quantity other than $E_{\gamma}$ is neglected in accord with the Brink hypothesis [21]. Experimental data from average resonance capture [22] and from ${ }^{3} \mathrm{He}$-induced reaction [17] seem to confirm at least the approximate validity of this hypothesis in the region of excitation energies below $S_{\mathrm{n}}$. From the above list of $E 1$ PSF models, only the SLO, KMF-T, and models based on HFB-QRPA microscopic calculations were assumed to follow the strict form of the Brink hypothesis. The remaining models predict a weak dependence of the PSF on the nuclear temperature of the final state $T_{\mathrm{f}}$ (which is equivalent to dependence on the excitation energy). 

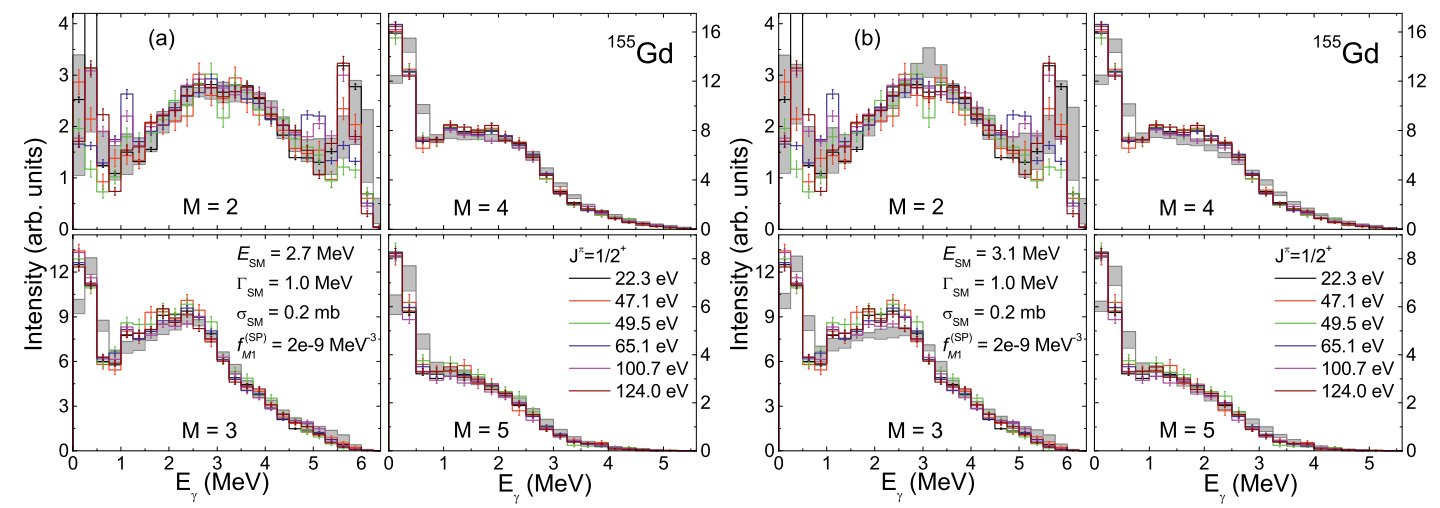

Figure 4. Illustration of the influence of different postulated energies of the SM on the predicted MSC spectra for ${ }^{155} \mathrm{Gd}$. The MGLO model (with $k_{0}=3$ ) was used for the $E 1$ PSF and the composite model $f_{M 1}^{(\mathrm{SM})}+f_{M 1}^{(\mathrm{SP})}+f_{M 1}^{(\mathrm{SF})}$ for the $M 1$ PSF. The parameters of the SM and the value of $f_{M 1}^{(\mathrm{SP})}$ are indicated in the figures together with the energies, spin $J$ and parity $\pi$ of the $s$-wave neutron resonances used in the analysis. The experimental spectra are the same in both parts of the figure. The gray band characterizes the result from simulations of 20 different NRs and has the width of two sigma - an average of +/- one sigma, see the text. The simulated MSC spectra with $E_{\mathrm{SM}}=2.7 \mathrm{MeV}$ (a) nicely reproduce the experimental data while for $E_{\mathrm{SM}}=3.1 \mathrm{MeV}$ (b) the description of the bump near $E_{\gamma}=2.5 \mathrm{MeV}$ is problematic as the bump has a different shape, especially for multiplicities $M=3$ and 4 .

\subsection{Magnetic dipole transitions}

Magnetic dipole $(M 1)$ transitions also play an important role in the decay of highly excited nuclear states below $S_{\mathrm{n}}$. In the mid 70's an isovector $M 1$ collective vibrational mode at $E_{\gamma} \approx 3 \mathrm{MeV}$ was predicted in deformed nuclei [23-25]. This mode, known as the scissors mode (SM), was observed for the first time for ground-state transitions in ${ }^{156} \mathrm{Gd}[26]$ in high-resolution electron inelastic scattering at low momentum transfer.

A systematic study of ground-state transitions for $E_{\gamma} \leq 4 \mathrm{MeV}$ in even-even rare-earth nuclei was performed with nuclear resonance fluorescence (NRF) experiments using the $\left(\gamma, \gamma^{\prime}\right)$ reaction [27]. The low density of $J=1$ states allowed observation of a majority of the dipole transition strength at these energies. In these experiments the total observed reduced $M 1$ strength $\sum B(M 1) \uparrow$ in the energy range $E_{\gamma} \approx 2.5-4.0 \mathrm{MeV}$ was proportional to the square of the nuclear deformation [28, 29] and for well deformed nuclei reached $\sum B(M 1) \uparrow \approx 3 \mu_{N}^{2}$. This strength is usually distributed over only a few states. All of the $M 1$ strength in this energy range was usually attributed to the SM.

In odd nuclei, the spacing between nuclear levels near the excitation energy $E_{\mathrm{x}}=3 \mathrm{MeV}$ is much smaller, which causes the fragmentation of the SM strength into many weak transitions. The limited experimental sensitivity prevents observation of all these transitions in the NRF experiments. This fact led to smaller observed SM strength in odd nuclei compared to even-even neighbors [30-33]. Attempts to estimate the missing strength in NRF reaction in odd nuclei were made using a fluctuation analysis [33, 34]. However, it seemed that this method did not work properly in some cases [33].

Later, the analysis of data in two-step cascades (TSC) following thermal neutron capture in ${ }^{162} \mathrm{Dy}$ suggested that a resonance-like structure of $M 1$ character with the strength of $\sum B(M 1) \uparrow \approx 6.2 \mu_{\mathrm{N}}^{2}$ couples not only to the ground state, but also to all levels in ${ }^{163} \mathrm{Dy}$ [35]. This observation gave strong support for the validity of the Brink hypothesis for the SM. A similar finding was corroborated 

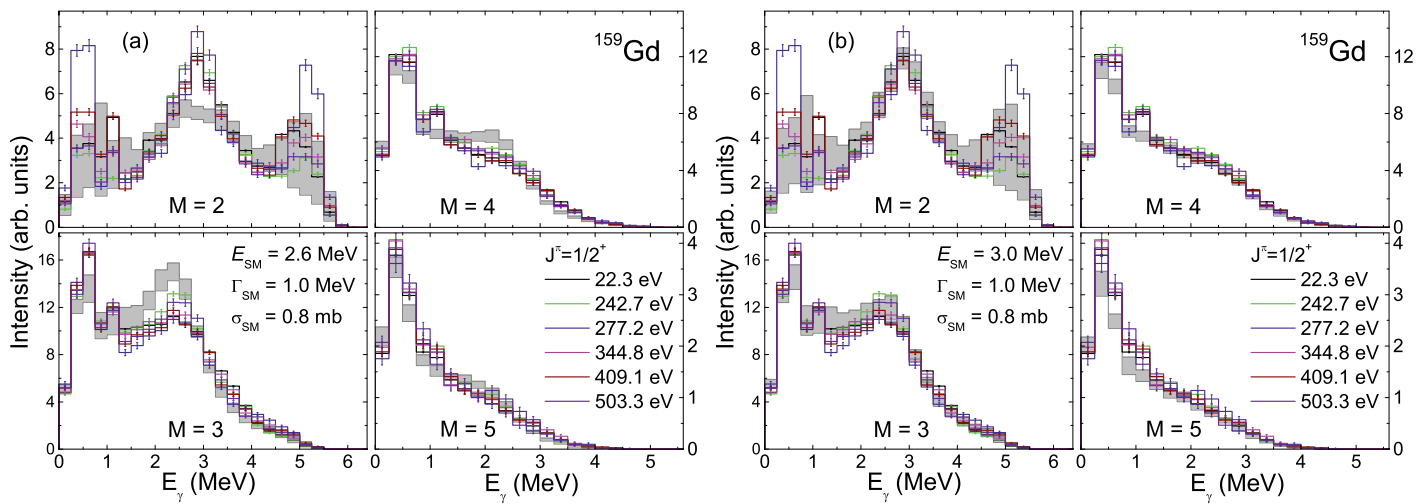

Figure 5. The same as in Fig. 4 but for ${ }^{159} \mathrm{Gd}$. In this case, the MGLO model (with $k_{0}=5$ ) was used for the $E 1 \mathrm{PSF}$. The MSC spectra predicted for $E_{\mathrm{SM}}=2.6 \mathrm{MeV}$ (a) - the energy that leads to reproduction of the Oslo data for Dy isotopes [16] - completely fail in describing the measured MSC spectra for multiplicities $M=2-4$, while the spectra for $E_{\mathrm{SM}}=3.0 \mathrm{MeV}$ (b) nicely agree with the experimental data.

by the TSC measurement of the ${ }^{159} \mathrm{~Tb}(\mathrm{n}, \gamma)$ reaction [36], and also by data on ${ }^{3} \mathrm{He}$-induced photon production [37].

The analysis of TSC spectra in Ref. [35] also indicated that the reduced SM strength in odd ${ }^{163}$ Dy $\left(\sum B(M 1) \uparrow \approx 6.2 \mu_{\mathrm{N}}^{2}\right)$ is higher compared to that deduced from $\left(\gamma, \gamma^{\prime}\right)$ measurements on neighboring even-even nuclei. Contrary to this, comparable strength of the SM was reported for ${ }^{3} \mathrm{He}$-induced reactions on ${ }^{160-164} \mathrm{Dy},{ }^{167} \mathrm{Er},{ }^{171,172} \mathrm{Yb}$ isotopes [16, 17, 19, 20]. Interestingly, data obtained from the same reaction yielded about 1.7 times lower strength of the SM in even-even ${ }^{166} \mathrm{Er}$ than in odd ${ }^{167} \operatorname{Er}[19]$.

The data from TSC measurements and ${ }^{3} \mathrm{He}$-induced reactions indicate that the SM is a resonancelike structure with a halfwidth of $\approx 0.7-1.5 \mathrm{MeV}$. The maximum of the resonance structure is close to $3 \mathrm{MeV}$ in the case of the ${ }^{163}$ Dy product [35], while it is slightly shifted down to about $2.7-2.8$ $\mathrm{MeV}$ in ${ }^{160} \mathrm{~Tb}$ [36] and even more in data from the ${ }^{3} \mathrm{He}$-induced reactions [16, 17, 19, 20].

The $M 1$ strength is likely more complex than just the contribution of the SM. Almost exclusively one of two models is used for the remaining part of the $M 1$ strength. In the spin-flip (SF) model the $f_{M 1}^{(\mathrm{SF})}\left(E_{\gamma}\right)$ is assumed to be a Lorentzian resonance function with the energy position at about $7 \mathrm{MeV}$ and a width of about $4 \mathrm{MeV}$ [12], while in the single-particle (SP) model $f_{M 1}^{(\mathrm{SP})}$ is a constant independent of $\gamma$-ray energy. In some cases a composite model for the $M 1$ strength, $f_{M 1}=f_{M 1}^{(\mathrm{SM})}+$ $f_{M 1}^{(\mathrm{SF})}+f_{M 1}^{(\mathrm{SP})}$, was used for the $M 1$ PSF. The absolute values of the SP and SF models were adjusted to obtain the ratio of $R=f_{E 1} / f_{M 1} \approx 7$ at $7 \mathrm{MeV}$ in our analysis. This value seems to be reasonably well determined in rare-earth nuclei from average resonance capture (ARC) experiments [22]. In models adopting the sum of SP and SF contributions, we varied $f_{M 1}^{(\mathrm{SP})}$ and adjusted the size of the SF contribution to reproduce the ratio $R$. The strict validity of the Brink hypothesis was assumed for the SP and SF $M 1$ models.

\subsection{Higher multipolarities}

In addition to dipole transitions, electric quadrupole $(E 2)$ transitions might also play a role in neutron resonance decay. In our analysis we either postulate the shape of the $f_{E 2}$ PSF as the standard 

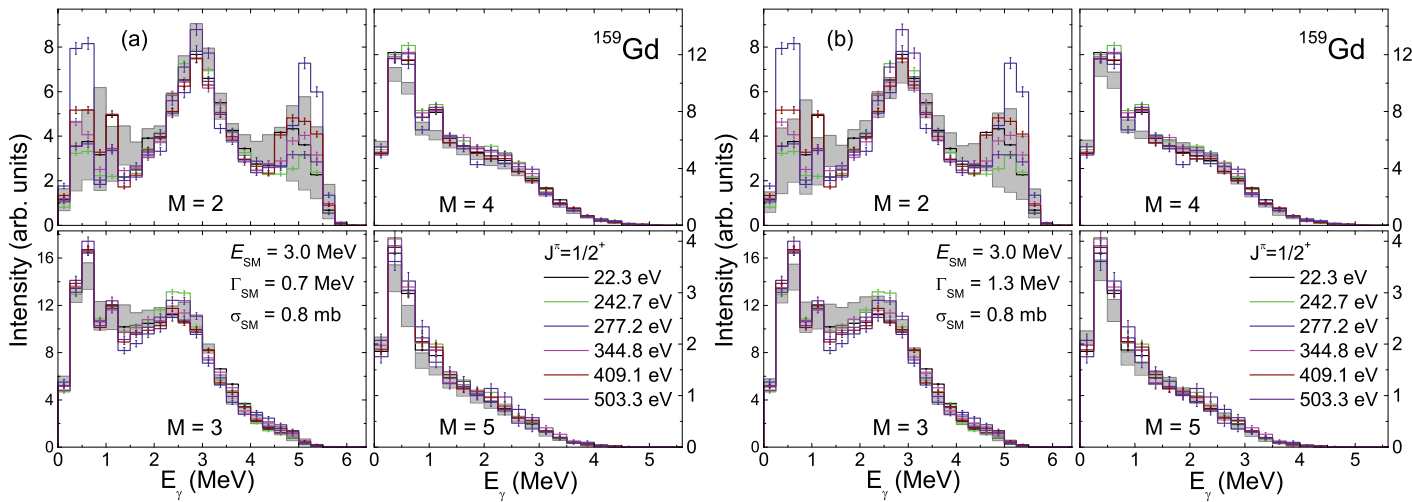

Figure 6. Illustration of the low sensitivity of predicted MSC spectra to different postulated widths of the SM for ${ }^{159} \mathrm{Gd}$. Experimental data are reasonably reproduced with $\Gamma_{\mathrm{SM}}=0.7 \mathrm{MeV}$ (a) as well as $\Gamma_{\mathrm{SM}}=1.3 \mathrm{MeV}$ (b). The MGLO model (with $k_{0}=5$ ) was used for the E1 PSF. For further description see the caption of Fig. 4.

Lorentzian form [38] or we adopt a single-particle model for the $E 2 \mathrm{PSF}$, in which $f_{E 2}^{(\mathrm{SP})}$ is a constant independent of the $\gamma$-ray energy. In our analysis the $E 2$ strength was taken to reproduce the ratio of partial radiation widths at about $7 \mathrm{MeV}$ measured in ARC experiments in even-even deformed nuclei, that is $\Gamma(E 1) / \Gamma(E 2) \geq 100[22]$.

The contributions of higher multipolarities, i.e., $M 2, E 3, \ldots$, are considered to be marginal in the decay of highly excited states of the compound nucleus and we completely neglect them in our simulations. This is justified by the fact that only a few very weak transitions of these types have been detected so far, mainly in the region of low excitations.

\subsection{Nuclear level density}

Two phenomenological NLD models given by closed-form formulas were tested: (i) the Back-Shifted Fermi Gas (BSFG), and (ii) the Constant-Temperature (CT) model [39]. There are two adjustable parameters in each of these models. Two different sets of parameters taken from papers of von Egidy and Bucurescu $[39,40]$ were tested. The spin distribution $f(J, \sigma)$ of the NLD for both of the models was adopted in the standard form $[12,39,40]$ with different expressions introduced for the spin-cutoff parameter $\sigma$ in Refs. $[39,40]$. The parity distribution function was usually considered in the simplest possible form $f(\pi)=1 / 2$ in our calculations.

In addition to these closed-form models, we also tested the microscopic NLD model based on the Hartree-Fock-Bogoljubov (HFB) method with the BSk14 effective Skyrme force [41]. The HFB microscopic NLDs are available as tables for different excitation energies, spins, and both parities. These tables can be found in the RIPL-3 database [12]. The NLD calculated in the HFB approach usually suffers from difficulties in reproducing the average neutron resonance spacing. In order to bring the calculations into agreement with experimental data, the tabulated HFB level densities are proposed to be normalized to reproduce a resonance spacing at the neutron separation energy [12].

Some of the PSF and NLD models tested in our simulations for ${ }^{159} \mathrm{Gd}$ isotope are shown in Fig. 3 . These models are compared with the experimental PSF and NLD values extracted by the Oslo method from the $\left({ }^{3} \mathrm{He}, \alpha\right)$ data on ${ }^{161} \mathrm{Dy}[16]$. 

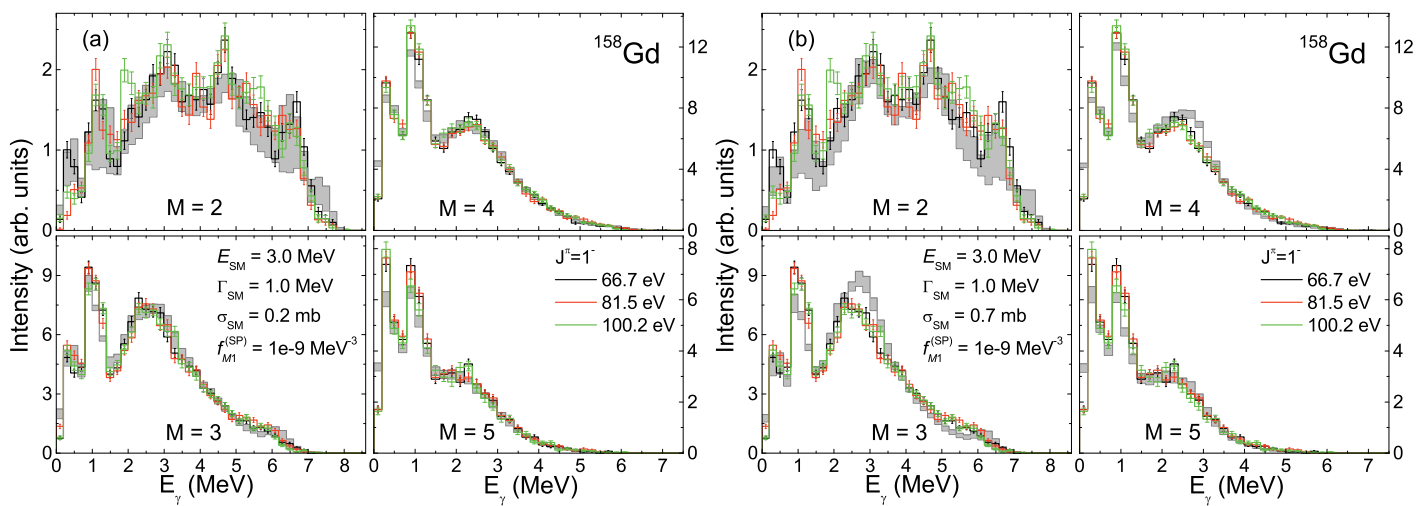

Figure 7. Illustration of the sensitivity of predicted MSC spectra to different postulated strength of the SM for $J^{\pi}=1^{-}$resonances of ${ }^{158} \mathrm{Gd}$ product. The SM parametrization in part (b), which does not reproduce experimental MSC spectra for $M=3$ and 4, nicely reproduces the MSC spectra in ${ }^{157,159} \mathrm{Gd}$. Contrary to this the SM parametrization in part (a) is unable to reproduce experimental MSC spectra in ${ }^{157,159} \mathrm{Gd}$, see Refs. [5, 6]. The MGLO model (with $k_{0}=2$ ) was used for E1 PSF. For further details see Fig. 4. Almost identical results were obtained for MSC spectra from $J^{\pi}=2^{-}$resonances of ${ }^{158} \mathrm{Gd}$ as well as for MSC spectra from resonances of both possible spins of ${ }^{156} \mathrm{Gd}$.

\section{Results from MSC spectra}

Several hundred model combinations of PSFs and NLD were simulated and compared with the experimental MSC spectra for each Gd isotope studied. To quantify precisely the agreement between the simulations and the experimental spectra extremely time-consuming simulations would be needed as the individual bins in MSC spectra are mutually correlated in a complicated way and the corresponding correlation matrix is not a priori known. As a consequence, the degree of agreement was checked only visually.

As mentioned in Sec. 4, DICEBOX simulations of nuclear electromagnetic decay correctly incorporate the Porter-Thomas fluctuations of partial radiation widths, as well as fluctuation properties of NLD. Results of individual NRs are together characterized by their mean and sigma. For the sake of clarity the standard confidence interval with a width of two sigma (the average value \pm one sigma) is plotted as a grey band in Figs. 4-8. The size of fluctuations among the experimental spectra for different resonances is reasonably well reproduced by the simulations which justifies applicability of the statistical model for description of the $\gamma$ decay of studied nuclei at energies below $S_{\mathrm{n}}$.

It was found that the influence of the E2 PSF on the shape of simulated MSC spectra is negligible. The constant $f_{E 2}^{(\mathrm{SP})}$ PSF function with the strength $f_{E 2}^{(\mathrm{SP})}=3-5 \times 10^{-11} \mathrm{MeV}^{-5}$ was usually used in simulations of the MSC spectra.

\subsection{Nuclear level density}

We were not able to reproduce experimental MSC spectra using the CT model of the NLD in combination with any of the tested PSF models for any Gd isotope. Simulations with the CT model give a clear shift toward lower values in the multiplicity distribution as compared to those obtained with the BSFG model. This feature might be expected from the energy dependence of NLD models shown in Fig. 3(b), where the ratio of levels available at very low excitation energy with respect to energies near 
3-5 MeV is higher than for the CT model. Such an effect suppresses transitions with lower $\gamma$ ray energies and leads to an evident preference for lower multiplicities in the CT model, in disagreement with the experimental results. On the other hand, for all studied nuclei we were able to find several models of PSFs which reproduce data in combination with the BSFG model. In the case of the NLD adopted from the HFB theoretical calculations, we obtained acceptable agreement with the experimental data only for ${ }^{153,155,158} \mathrm{Gd}$ products.

\subsection{Electric dipole PSF}

It appears that the strong influence of the $M 1 \mathrm{SM}$ on the $\gamma$ decay of studied Gd nuclei, discussed in Sec. 5.3, significantly suppresses our sensitivity to the $E 1$ PSF. From the comparison of the shapes of experimental and simulated MSC spectra, we are not able to identify one particular E1 PSF model as the most acceptable one.

In all Gd isotopes we obtained an acceptable agreement between simulated and experimental MSC spectra for the GH, KMF, KMF-T (with $T_{\mathrm{f}} \approx 0.25-0.35 \mathrm{MeV}$ ), and MGLO (with variable $k_{0}$ ) models. The allowed values of the $k_{0}$ parameter in the MGLO model seem to increase with the mass of the isotope from about $1.5-2.0$ (for ${ }^{153} \mathrm{Gd}$ ) to about $4.0-5.0$ (for ${ }^{157,159} \mathrm{Gd}$ ). A few models, specifically the SLO, MLO1, MLO2 and MLO3 ones, were acceptable only for specific isotopes. On the other hand, we completely failed using the GFL, EGLO, as well as the microscopic E1 PSF models in all nuclei. The list of acceptable models given above indicates that we were unable to confirm or reject the necessity of a temperature dependence of the $E 1 \mathrm{PSF}$.

\subsection{Magnetic dipole PSF}

The MSC spectra for all studied Gd isotopes clearly show a pronounced peak (in odd nuclei) or double-peak (in even-even nuclei) structure present in the middle of the $M=2$ spectrum. A clear bump is present also between 2 and $3 \mathrm{MeV}$ in the $M=3 \mathrm{MSC}$ spectrum. These maxima in the MSC spectra indicate the presence of a resonance structure in a PSF. Indeed, all models that do not include a resonance near $3 \mathrm{MeV}$ in a PSF are unable to reproduce the observed shapes of the MSC spectra in all Gd isotopes. This effect is more pronounced in heavier odd ${ }^{157,159} \mathrm{Gd}$ isotopes, where the observed peak structure is much stronger than in the other nuclei studied.

We found that the resonance cannot be purely of $E 1$ character, see Refs. [6, 46, 47]. The sensitivity of the predicted MSC spectra to the type of transitions comes from the fact that $\gamma$ cascades start from $s$-wave resonances which have a specific parity. As spin and parity selection rules for different types and multipolarities of transitions are fully taken into account in the simulations, the multiplicity distribution as well as the shape of the MSC spectra for different multiplicities are sensitive to the ratio of $f_{E 1} / f_{M 1}$ below $S_{\mathrm{n}}$. We need $f_{E 1} / f_{M 1} \approx 1$ at $E_{\gamma}$ between about 2 and $4 \mathrm{MeV}$. If the resonance structure at $3 \mathrm{MeV}$ was in the $E 1 \mathrm{PSF}$, the ratio would be $f_{E 1} / f_{M 1} \gg 1$ and the peak structure observed in the $M=2$ MSC spectra would not be reproduced by the simulations, as one of the two $\gamma$ rays in the two-step cascade has to be of $M 1$ (or E2) character in all Gd isotopes. On the other hand, a weak contribution of the $E 1$ strength to the resonance structure cannot be fully excluded.

In reality, we are unable to unambiguously distinguish between the $M 1$ and $E 2$ character of the resonance as the MSC spectra calculated with both multipolarities often have a very similar shape. This is due to the similar spin and parity selection rules for these two types of transitions. Nevertheless, simulations with a resonance structure postulated in the E2 PSF yielded slightly worse agreement in all tested cases. This fact combined with the expectation of the presence of the $M 1 \mathrm{SM}$ near $3 \mathrm{MeV}$ in heavy well-deformed nuclei and no prediction of strong transitions of $E 2$ character with $E_{\gamma} \approx 3 \mathrm{MeV}$ 
lead us to identify the structure responsible for the peaks observed in the experimental MSC spectra with the $M 1$ scissors mode.

The MSC spectra are sensitive to the parameters of the SM postulated in the M1 PSF. In all cases a Lorentzian shape was assumed with three independent parameters, energy $E_{\mathrm{SM}}$, width $\Gamma_{\mathrm{SM}}$, and the maximum cross section $\sigma_{\mathrm{SM}}$ of the SM. The minimum and maximum values of these parameters, together with the corresponding values of the reduced transition probability $\sum B(M 1) \uparrow$, that lead to the agreement between the shape of experimental and simulated MSC spectra, can be found in Ref. [6]. The comparison of simulated MSC spectra with experimental ones for models that nicely reproduce the measured spectra can be found in Refs. [6, 46-48]. In this contribution we would rather illustrate the sensitivity of simulations to the change in different SM parameters.

We found that the energy of the SM, $E_{\mathrm{SM}}$, must be very close to $3 \mathrm{MeV}$ in ${ }^{153,156,157,158,159} \mathrm{Gd}$ but it is shifted to lower values in ${ }^{155} \mathrm{Gd}$. This is illustrated in Figs. 4 and 5 for ${ }^{155} \mathrm{Gd}$ and ${ }^{159} \mathrm{Gd}$, respectively. The energy $E_{\mathrm{SM}}=2.7 \mathrm{MeV}$ in ${ }^{155} \mathrm{Gd}$ is very close to the value obtained from the analysis of data from ${ }^{3} \mathrm{He}$-induced reactions in the Dy isotopes [16]. As illustrated in Fig. 5, our data for the remaining isotopes are in contradiction with the position of the SM measured in Dy.

The damping width of the SM, $\Gamma_{\mathrm{SM}}$, can reach a rather broad range of values between about 0.7 and $1.3 \mathrm{MeV}$ in all studied isotopes, see Ref. [6]. Restricted sensitivity to $\Gamma_{\mathrm{SM}}$ is illustrated in Fig. 6 for ${ }^{159} \mathrm{Gd}$.

Both the $E_{\mathrm{SM}}$ and $\Gamma_{\mathrm{SM}}$ are almost independent of the $E 1$ PSF model used in the simulations. In addition, the allowed values of $\sigma_{\mathrm{SM}}$ are only slightly correlated with $E_{\mathrm{SM}}$ and $\Gamma_{\mathrm{SM}}$. On the other hand, one can expect a scaling of the size of the bump near $E_{\gamma}=3 \mathrm{MeV}$ (which can be described by $\sigma_{\mathrm{SM}}$ ) produced in simulations of MSC spectra with the absolute size of the E1 PSF model. Such a dependence is really observed. It means that the allowed values of $\sigma_{\mathrm{SM}}$ in combination with the $\mathrm{KMF}$, GH, or MGLO (with $k_{0} \approx 1.5$ ) models are smaller than with the SLO, MLO2, or MGLO(with $k_{0} \approx 4-5$ ) models. For detailed discussion of this effect, see Ref. [6].

We have found that the values of $\sigma_{\mathrm{SM}}$ obtained for even-even ${ }^{156,158} \mathrm{Gd}$, odd ${ }^{153} \mathrm{Gd}$, and partly also for ${ }^{155} \mathrm{Gd}$, are significantly lower than those for ${ }^{157,159} \mathrm{Gd}$ - the strength in ${ }^{157,159} \mathrm{Gd}$ isotopes is about three times higher than in ${ }^{156,158} \mathrm{Gd}$ and ${ }^{153} \mathrm{Gd}$ and approximately two times higher than in ${ }^{155} \mathrm{Gd}$ nucleus. This effect persists with a fixed $E 1$ PSF model. The significant difference in $\sigma_{\mathrm{SM}}$ is apparent for ${ }^{155} \mathrm{Gd}$ and ${ }^{158} \mathrm{Gd}$ from Figs. 7 and 8 , respectively. In these figures, the higher values of $\sigma_{\mathrm{SM}}$ correspond to those needed for reproduction of MSC spectra in ${ }^{157,159} \mathrm{Gd}$. We have no explanation of this odd-even difference in the SM strength at the moment.

In reality, more complex model for the $M 1$ PSF than just the SM seem to be needed. In particular, a "non-resonant" single particle $M 1$ component $f_{M 1}^{(\mathrm{SP})} \approx(1-2.5) \times 10^{-9} \mathrm{MeV}^{-3}$ had to be added to the $M 1$ PSF to reach a reasonable reproduction of experimental MSC spectra in the ${ }^{153,155,156,158} \mathrm{Gd}$ isotopes $[6,48]$. On the other hand, we are virtually insensitive to the exact parametrization of the spin-flip PSF $f_{M 1}^{(\mathrm{SF})}$ postulated in our simulations.

To reach reasonable agreement between simulated and experimental MSC spectra, the SM has to be postulated on all excited levels that are accessible in the $\gamma$ decay of the studied Gd isotopes. More precisely, as illustrated in Refs. [5, 6], we need the SM to be built on all levels up to at least 5 or 6 $\mathrm{MeV}$ of excitation energy. For higher excitation energies the sensitivity of our method decreases as the SM resonance structure built on levels above 4-5 MeV in odd and 6-7 MeV in even-even studied $\mathrm{Gd}$ nuclei does not influence the decay of neutron resonances in these isotopes. Our sensitivity is also much better for ${ }^{157,159} \mathrm{Gd}$ products, where the SM is two or three times stronger than in the other Gd isotopes studied. We cannot fully guarantee that the parameters of the SM are completely independent of the excitation energy. However, we tried to change systematically - usually linearly the SM parameters $\left(E_{\mathrm{SM}}, \Gamma_{\mathrm{SM}}, \sigma_{\mathrm{SM}}\right)$ with the excitation energy and we found that the best agreement 

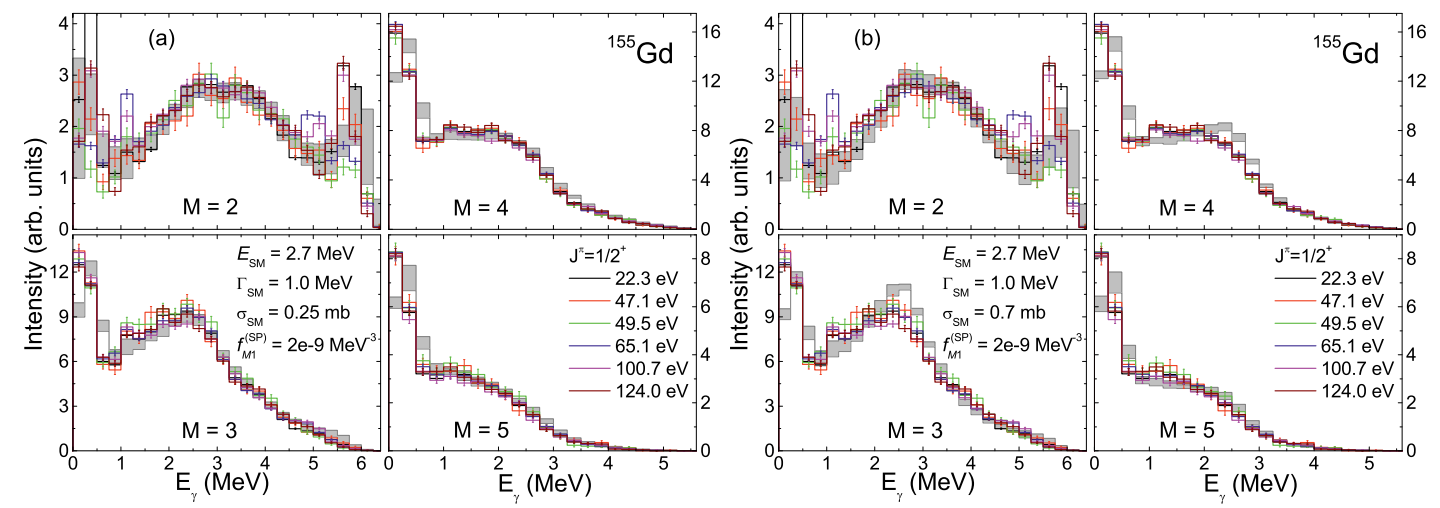

Figure 8. The same as in Fig. 7 but for MSC spectra in ${ }^{155} \mathrm{Gd}$. The MGLO model (with $k_{0}=3$ ) was used for the E1 PSF. For further details see Fig. 4.

for all nuclei is obtained with the SM following exactly the Brink hypothesis. The SM is thus a resonance structure present in the M1 PSF following the Brink hypothesis [21].

\section{Comparison with other experimental data}

Several measurements provide information on the PSFs in the low-energy tail of the GDER in the region of $A \approx 150-175$. In addition to the outcome of the analysis of MSC spectra presented in this contribution, there are other data from (n, $\gamma$ ) reactions: (i) the absolute values of PSFs were obtained from the intensities of primary transitions following the resonance neutron capture in ${ }^{154,156,158} \mathrm{Gd}$ target nuclei [44, 45], (ii) two-step cascades (TSC) measurements on ${ }^{162} \mathrm{Dy}$ [35] and ${ }^{159} \mathrm{~Tb}$ [36] targets provided information on the PSFs with the emphasis put on the properties of the scissors mode, (iii) information about the shapes of PSFs near $S_{\mathrm{n}}$ was also obtained from the average resonance neutron capture experiments on ${ }^{156,158} \mathrm{Gd}$ product nuclei $[14,22]$ and (iv) the experimental values of the total radiation width of $s$-wave neutron resonances are available for all stable isotopes $[12,50]$.

Additional information on PSFs comes from ${ }^{3} \mathrm{He}$-induced reactions measured for several rareearth nuclei at the Oslo Cyclotron Laboratory [16-20]. Finally, data on the reduced $M 1$ strength $\sum B(M 1) \uparrow$ of the ground-state transitions are available from $\left(\gamma, \gamma^{\prime}\right)$ measurements for all stable eveneven rare-earth nuclei [27] and several odd rare-earth nuclei [30, 31, 33, 34, 49]. In the case of a couple of even-even nuclei, data from $\left(\mathrm{e}, \mathrm{e}^{\prime}\right)$ experiments confirm the results from $\left(\gamma, \gamma^{\prime}\right)$ experiments $[26,51]$.

\subsection{Total radiation width}

The DICEBOX code can simulate the $\gamma$ cascades which are used for production of the MSC spectra, as well as any other quantity related to the radiative neutron capture at low neutron energies. Almost all of these quantities depend only on the energy-dependent relative ratios of the PSFs for different types and multipolarities of transitions. One of the quantities that depends on the absolute values of the PSFs and the NLD and is easily comparable to experimentally measured values is the total radiation width of $s$-wave neutron resonances $\Gamma_{\gamma}$. The value of $\Gamma_{\gamma}$ is a simple sum of contributions of different types and multipolarities of transitions, in our case $\Gamma_{\gamma}=\Gamma_{\gamma}^{(E 1)}+\Gamma_{\gamma}^{(M 1)}+\Gamma_{\gamma}^{(E 2)}$. Contributions of different 


\section{$\mathrm{CNR} * 13$}

Table 1. Contributions of different $E 1$ PSF models and the SM to the total radiation widths of $s$-wave neutron resonances of studied Gd nuclei. The isotopes correspond to the product nuclei in (n, $\gamma)$ reaction. The energy of the $\mathrm{SM}$ was $E_{\mathrm{SM}}=3.0 \mathrm{MeV}$ for all isotopes except ${ }^{155} \mathrm{Gd}$, where $E_{\mathrm{SM}}=2.6 \mathrm{MeV}$. The SM width was $\Gamma_{\mathrm{SM}}=1.0 \mathrm{MeV}$ in all cases and the maximum cross section was $\sigma_{\mathrm{SM}}=0.2,0.3,0.2,0.7,0.2,0.7 \mathrm{mb}$ for $153,155,156,157,158,159 \mathrm{Gd}$, respectively. Uncertainties in the simulated values of $\Gamma_{\gamma}$ originate from different NRs.

\begin{tabular}{|c|c|c|c|c|c|c|}
\hline \hline$\Gamma_{\gamma}^{(E I)}$ or $\Gamma_{\gamma}^{(M \mathrm{I})}(\mathrm{meV})$ & ${ }^{153} \mathrm{Gd}$ & ${ }^{155} \mathrm{Gd}$ & ${ }^{156} \mathrm{Gd}$ & ${ }^{157} \mathrm{Gd}$ & ${ }^{158} \mathrm{Gd}$ & ${ }^{159} \mathrm{Gd}$ \\
\hline SLO & $128(4)$ & $127(3)$ & $209(5)$ & $143(6)$ & $183(5)$ & $140(9)$ \\
KMF & $33(1)$ & $35(1)$ & $65(1)$ & $41(2)$ & $56(1)$ & $38(3)$ \\
MGLO $\left(k_{0}=2\right)$ & $34(1)$ & $37(1)$ & $72(2)$ & $44(2)$ & $62(2)$ & $40(3)$ \\
MGLO $\left(k_{0}=3\right)$ & $44(1)$ & $48(2)$ & $95(2)$ & $56(2)$ & $81(2)$ & $50(3)$ \\
MGLO $\left(k_{0}=4\right)$ & $55(1)$ & $60(2)$ & $117(3)$ & $69(2)$ & $100(3)$ & $62(4)$ \\
MGLO $\left(k_{0}=5\right)$ & $69(2)$ & $71(2)$ & $139(4)$ & $83(4)$ & $118(4)$ & $77(6)$ \\
MLO1 & $60(2)$ & $61(2)$ & $134(3)$ & $69(3)$ & $110(3)$ & $63(4)$ \\
MLO2 & $81(2)$ & $81(2)$ & $143(3)$ & $92(4)$ & $123(3)$ & $88(6)$ \\
MLO3 & $70(2)$ & $69(2)$ & $116(3)$ & $77(3)$ & $99(3)$ & $74(5)$ \\
SM & $6(1)$ & $12(1)$ & $9(1)$ & $24(1)$ & $9(1)$ & $24(2)$ \\
Experiment [12] & $54(5)$ & $75(6)$ & $108(10)$ & $88(12)$ & $97(10)$ & $105(10)$ \\
Experiment [50] & $55(3)$ & $74(3)$ & $110(3)$ & $88(12)$ & $99(6)$ & $90(6)$ \\
\hline \hline
\end{tabular}

E1 PSF models to $\Gamma_{\gamma}$ are listed in Tab. 1. It appears that the fraction of $\Gamma_{\gamma}$ that originates from the E2 PSF is less than 5\%, the part from the SF M1 PSF is up to $10 \%$ for ${ }^{153,155} \mathrm{Gd}$ and below $5 \%$ for the other Gd nuclei studied. As the single-particle component of the M1 PSF contributes up to about $17 \%$ to $\Gamma_{\gamma}$, the remaining $75-90 \%$ of $\Gamma_{\gamma}$ comes from the $E 1$ PSF and the $M 1$ SM strength. The value of $\Gamma_{\gamma}$ also strongly depends on the model of the NLD. As discussed above only the BSFG model of the NLD leads to the reproduction of experimental spectra in all studied Gd nuclei. Therefore further discussion assumes the use of this NLD model.

The requirement on the reproduction of $\Gamma_{\gamma}$ can serve as an additional constraint on the acceptability of the PSFs models. For instance, the absolute size of $f_{E 1}$ for the SLO model in the energy region of the SM is much higher than in other E1 PSF models. As discussed above, this means that the reproduction of experimental MSC spectra requires a much higher strength of the SM (and thus also the contribution of the SM to $\Gamma_{\gamma}$ ) than for all other $E 1$ PSF models. In odd Gd isotopes, where the SLO model could reproduce the shape of MSC spectra, we would need $\sigma_{\mathrm{SM}}=0.6-0.8 \mathrm{mb}$ and $\sigma_{\mathrm{SM}}=1.6-2.0 \mathrm{mb}$ for the ${ }^{153,155} \mathrm{Gd}$ and ${ }^{157,159} \mathrm{Gd}$ isotopes, respectively. As a consequence, the value of $\Gamma_{\gamma}$ for the model which would reproduce the MSC spectra would be at least a factor two higher than the experimental value. We can thus definitely exclude the SLO model for the E1 PSF in these nuclei. We should add here that in even-even Gd products the SLO model reproduces neither the experimental values of $\Gamma_{\gamma}$ nor the shape of the measured MSC spectra.

As discussed already in Ref. [6], the experimental value of $\Gamma_{\gamma}$ significantly increases with the mass of the odd Gd isotopes, see Tab. 1. On the other hand, the calculations performed with the same $E 1$ PSF model lead to very similar values of $\Gamma_{\gamma}$ in all odd Gd isotopes. The predicted difference in $\Gamma_{\gamma}$ is thus mainly due to different SM contributions. But the SM alone can be responsible for less than a half of the increase of the experimental $\Gamma_{\gamma}$ value in odd Gd isotopes. This fact indicates that the $E 1 \mathrm{PSF}$ is responsible for a remaining part of this increase, which further implies that there is no universal parameter-free $E 1 \mathrm{PSF}$ model in the chain of odd Gd isotopes. The only tested $E 1$ PSF model that is able to reproduce the shape of the experimental MSC spectra and the experimental $\Gamma_{\gamma}$ 
values simultaneously in all Gd isotopes is the MGLO model. The adjustment of the free parameter $k_{0}$ introduced in the MGLO $E 1$ PSF model is able to mimic the anticipated change of the E1 PSF. The parameter has to vary from $k_{0} \approx 2$ for ${ }^{153} \mathrm{Gd}$, to $k_{0} \approx 4-5$ for ${ }^{159} \mathrm{Gd}$.

\subsection{Intensities of $E 1$ primary transitions}

Additional data on the $E 1$ PSF come from the measurement of intensities of primary $E 1$ transitions following the capture of slow neutrons in the ${ }^{154,156,158} \mathrm{Gd}$ isotopes $[44,45]$. Experimental data, which are shown in Fig. 3(a) indicate an increase of the $E 1$ PSF at $E_{\gamma}=5-7 \mathrm{MeV}$ with the mass of the isotope. These data correspond perfectly to our finding about the $E 1$ PSF discussed above. We would like to point out here that the $E 1$ PSF should match the Lorentzian shape at energies above $S_{\mathrm{n}}$. This condition requires a rather steep increase of the $E 1 \mathrm{PSF}$ at $E_{\gamma} \approx 5-9 \mathrm{MeV}$ in lighter Gd isotopes.

\subsection{Scissors mode}

The values of the SM parameters obtained from our analysis of the MSC spectra of Gd isotopes can be compared to values deduced from TSC experiments, ${ }^{3} \mathrm{He}$-induced reactions and $\left(\gamma, \gamma^{\prime}\right)$ experiments.

As discussed above, there is a discrepancy in the extracted energy of the SM. The position of the mode which comes from ${ }^{3} \mathrm{He}$-induced reactions is usually too low to be acceptable for description of the MSC spectra. The energy which comes from our analysis of MSC spectra perfectly agrees with results from $\left(\gamma, \gamma^{\prime}\right)$ experiments for the even-even rare-earth nuclei. We have no explanation for the difference in position deduced from different experimental techniques at the moment.

The damping width of the $\mathrm{SM}$ is $\Gamma_{\mathrm{SM}} \approx 1.0 \mathrm{MeV}$ in all studied Gd isotopes. This value is comparable to results from previous TSC experiments $[35,36]$ as well as to data from ${ }^{3} \mathrm{He}$-induced reactions $[16,17,19,20]$. For completeness, we should notice that it seems to be difficult to say anything about the width of the SM from $\left(\gamma, \gamma^{\prime}\right)$ experiments. In even-even nuclei, the observed data from $\left(\gamma, \gamma^{\prime}\right)$ indicate that the SM is fragmented into only a few states that seem to be concentrated in a rather narrow region of less than half an $\mathrm{MeV}$ but the observed pattern in odd nuclei varies significantly.

Comparison of the strength of the SM from different experiments is rather difficult. Specifically, from the $\left(\gamma, \gamma^{\prime}\right)$ measurements one knows only the total $M 1$ reduced strength given by a sum of the strengths of individual $M 1$ transitions observed for the even-even rare-earth nuclei in the restricted energy interval $E_{\gamma}=2.7-3.7 \mathrm{MeV}$. In addition, the $\left(\gamma, \gamma^{\prime}\right)$ data on odd rare-earth nuclei must be nontrivially corrected for the unobserved strength and the result depends on the way the correction is performed [33, 34, 52]. Data from the Oslo method provide information on the SM and SF components of the M1 PSF [16-20], while our data indicate that the total $M 1$ strength consists of contributions from the SM, SF and SP components of the M1 PSF.

Therefore we can compare, for instance, the data from the $\left(\gamma, \gamma^{\prime}\right)$ experiments with the sum of SM, SF and SP components in our case, or the sum of SM and SF components from the Oslo method, integrated over the energy range $E_{\gamma}=2.7-3.7 \mathrm{MeV}$. Or we can compare the pure SM strength, $\sum B_{\text {tot }}(\mathrm{SM}) \uparrow$, obtained from the analysis of MSC spectra and from the Oslo method integrated over the whole energy range assuming a Lorentzian shape for the SM. The comparison of the latter quantity from different reactions for all nuclei as a function of the fractional number $P=N_{\mathrm{p}} N_{\mathrm{n}} /\left(N_{\mathrm{p}}+N_{\mathrm{n}}\right)$, where $N_{\mathrm{n}}$ and $N_{\mathrm{p}}$ is the number of valence neutrons and protons, respectively, see also Refs. [53-55], is shown in Fig. 9. The total $M 1$ strength observed in $\left(\gamma, \gamma^{\prime}\right)$ experiments at energies between 2.7 and 3.7 MeV was considered to be from the SM for the data plotted in the Fig 9. A comparison of the SM strength defined in a different way as a function of the neutron number $N$ or the square of the nuclear deformation parameter $\beta_{2}$ is discussed in detail in Refs. [6, 48, 56]. 


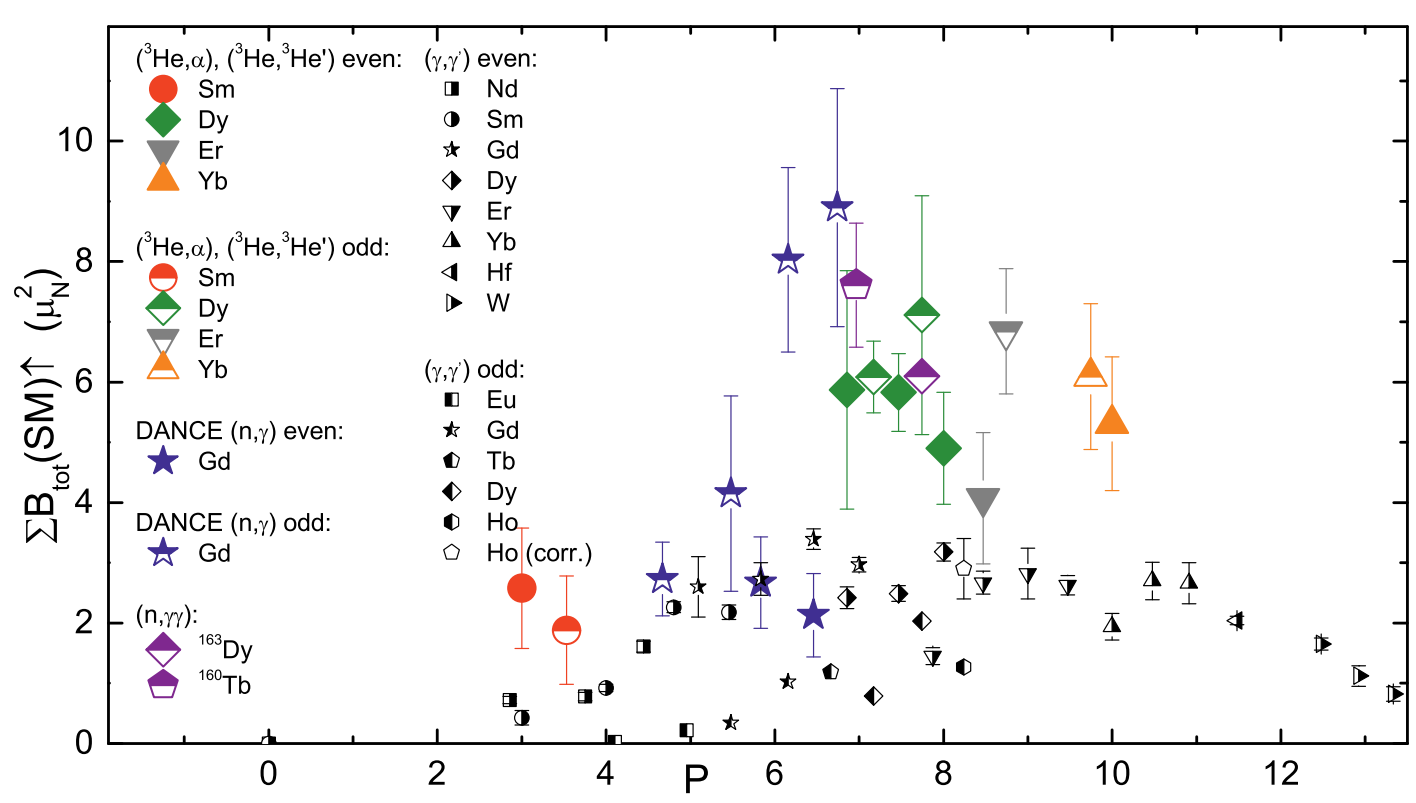

Figure 9. Comparison of the total reduced SM strength $\sum B_{\mathrm{tot}}(\mathrm{SM}) \uparrow$ for rare-earth nuclei as a function of the fractional number $P$ which is defined in the text. Our results $[6,46,47]$ are plotted together with the NRF data [27, 33], the data from the ${ }^{3} \mathrm{He}$-induced reactions [16-20] and the values obtained in the TSC measurements [35, 36]. The corrected value for ${ }^{165} \mathrm{Ho}$ was obtained from the fluctuation analysis [33].

As discussed above, we see a significant difference between the SM strength in well-deformed odd and even-even Gd isotopes. Our results for ${ }^{157,159} \mathrm{Gd}$ are in very good accord with the Oslo data for odd $\mathrm{Dy}, \mathrm{Er}$ and $\mathrm{Yb}$ isotopes, and with the values from the TSC experiments on ${ }^{163} \mathrm{Dy}$ and ${ }^{160} \mathrm{~Tb}$. However, we observe significantly smaller SM strength in even-even ${ }^{156,158} \mathrm{Gd}$ product nuclei compared to neighboring odd ${ }^{157,159} \mathrm{Gd}$ isotopes. Such an odd-even effect seemed to be observed also for the ${ }^{166,167} \mathrm{Er}$ isotopes studied in Oslo [19], while virtually no odd-even asymmetry was measured in ${ }^{3} \mathrm{He}$-induced reactions for Dy and $\mathrm{Yb}$ isotopes. Unfortunately, the SM parameters, and thus also the SM strength, determined from the Oslo method strongly depend on the nuclear temperature assumed for the $E 1$ PSF model used in the fit to the Oslo experimental data. The total SM strength is directly proportional to the $\Gamma_{\mathrm{SM}} \times \sigma_{\mathrm{SM}}$ product, which can change by a factor of more than two for thee different temperatures used in the fit to the same data [16]. Oslo values presented for the Dy isotopes correspond to the KMF-T model of the $E 1 \mathrm{PSF}$ with the temperature $T=0.3 \mathrm{MeV}$.

\section{Summary}

Measurements of $\gamma$-ray spectra from strong isolated $s$-wave neutron resonances on isotopically enriched ${ }^{152,154-158}$ Gd targets were performed using the DANCE detector array installed at the LANSCE spallation neutron source. The MSC spectra obtained from these resonances were used to test the validity of various PSF and NLD models. Taking into account additional available information on PSFs and the NLD, the main results of our analysis can be summarized as: (i) The energy dependence of the NLD is well described with the BSFG model. The energy dependence predicted by the CT model is highly unlikely. (ii) The MGLO model with a variable parameter $k_{0}$ is the only tested $E 1$ 
PSF model that simultaneously reproduces the shapes of the measured MSC spectra and the experimental values of total radiation widths for all studied Gd nuclei. The validity of this model is also supported by the data on intensities of primary transitions from (n, $\gamma$ ) reactions. (iii) The experimental total radiation widths of the $s$-wave neutron resonances clearly show that the $E 1$ PSF in the studied Gd nuclei cannot be described by the Lorentzian extrapolation of the GDER. (iv) The shapes of the experimental MSC spectra indicate the presence of a resonance structure in the M1 PSF near $3 \mathrm{MeV}$, that can be described by a Lorentzian curve. This structure was identified with the scissors mode. To reproduce the experimental spectra, the SM has to be built on all excited levels of the studied nuclei. The total reduced SM strength $\sum B_{\text {tot }}(\mathrm{SM}) \uparrow$ observed in odd Gd nuclei increases with the mass number. However, its dependence on the nuclear deformation, see Ref. [56], seems to be at variance with the $\beta_{2}^{2}$ dependence observed in $\left(\gamma, \gamma^{\prime}\right)$ measurements on even-even rare-earth nuclei [27-29]. (v) The $\mathrm{SM}$ observed in the ${ }^{157,159} \mathrm{Gd}$ isotopes is significantly stronger than that in the even-even ${ }^{156,158} \mathrm{Gd}$ nuclei. Similar odd-even staggering seems to be present in Oslo data for the ${ }^{166,167} \mathrm{Er}$ isotopes, while no odd-even asymmetry was observed in the Oslo results for Dy and Yb nuclei.

\section{Acknowledgments}

This work was supported in part by the U. S. Department of Energy Grants No. DE-NA0001784 and No. DE-FG02-97-ER41042. This work benefited from the use of the LANSCE accelerator and was performed under the auspices of the U. S. Department of Energy at Los Alamos National Laboratory by the Los Alamos National Security, LLC under contract No. DE-AC52-06NA25396 and LLNL contract No. DE-AC52-07NA27344. It was also supported by the research plan MSM 0021620859 of the Ministry of Education of the Czech Republic, and grant No. 13-07117S of the Czech Science Foundation.

\section{References}

[1] M. Heil et al., Nucl. Instrum. Methods Phys. Res. A 459, 229 (2001).

[2] R. Reifarth et al., Nucl. Instrum. Methods Phys. Res. A 531, 530 (2004).

[3] P. W. Lisowski and K. F. Schoenberg, Nucl. Instrum. Methods Phys. Res. A 562, 910 (2006).

[4] J. M. Wouters et al., IEEE Transactions on Nuclear Science 53, 880 (2006).

[5] J. Kroll et al., EPJ Web of Conferences 21, 04005 (2012).

[6] J. Kroll et al., Phys. Rev. C 88, 034317 (2013).

[7] F. Bečv ař, Nucl. Instrum. Methods Phys. Res. A 417, 434 (1998).

[8] M. Jandel et al., Nucl. Instrum. Methods Phys. Res. B 261, 1117 (2007).

[9] C. E. Porter and R. G. Thomas, Phys. Rev. 104, 483 (1956).

[10] S. G. Kadmenskij et al., Yad. Fiz. 37, 277 (1983); Sov. J. Nucl. Phys. 37, 165 (1983).

[11] J. Kopecky et al., Phys. Rec. C 47, 312 (1993).

[12] R. Capote et al., Nuclear Data Sheets 110, 3107 (2009).

[13] S. Goriely, Phys. Lett. B 436, 10 (1998).

[14] S. F. Mughabghab and C. L. Dunford, Phys. Lett. B 487, 155 (2000).

[15] V. A. Plujko et al., in Proc. 11th Int. Symp. Capt. Gamma-Ray Spectr. and Rel. Topics, Pruhonice near Prague, Czech Republic, World Scientific, Singapore, pp. 424 (2003).

[16] M. Guttormsen et al., Phys. Rev. C 68, 064306 (2003).

[17] H. T. Nyhus et al., Phys. Rev. C 81, 024325 (2010).

[18] S. Siem et al., Phys. Rev. C 65, 044318 (2002). 


\section{CNR*13}

[19] E. Melby et al., Phys. Rev. C 63, 044309 (2001).

[20] A. Voinov et al., Phys. Rev. C 63, 044313 (2001).

[21] D. M. Brink, Ph.D. Thesis, Oxford University, (1955).

[22] L. M. Bollinger and G. E. Thomas, Phys. Rev. C 2, 1951 (1970).

[23] R. R. Hilton, talk at International Conference on Nuclear Structure, JINR Dubna, (unpublished) (1976).

[24] N. Lo Iudice and F. Palumbo, Phys. Rev. Lett. 41, 1532 (1978).

[25] F. Iachello, Nucl. Phys. A 358, 89c (1981).

[26] D. A. Bohle et al., Phys. Lett. B 137, 27 (1984).

[27] U. Kneissl et al., Prog. Part. Nucl. Phys. 37, 349 (1996).

[28] W. Ziegler et al., Phys. Rev. Lett. 65, 2515 (1990).

[29] J. Margraf et al., Phys. Rev. C 47, 1474 (1993).

[30] J. Margraf et al., Phys. Rev. C 52, 2429 (1995).

[31] A. Nord et al., Phys. Rev. C 54, 2287 (1996).

[32] J. Enders et al., Phys. Rev. Lett. 79, 2010 (1997).

[33] A. Nord et al., Phys. Rev. C 67, 034307 (2003).

[34] N. Huxel et al., Nucl. Phys. A 645, 239 (1999).

[35] M. Krtička et al., Phys. Rev. Lett. 92, 172501 (2004).

[36] J. Kroll et al., Int. Jour. Mod. Phys. E, Vol. 20, Num. 2, 526 (2011).

[37] A. Schiller et al., Phys. Lett. B 633, 225 (2006).

[38] J. Kopecky and M. Uhl, Phys. Rev. C 41, 1941 (1990).

[39] T. von Egidy and D. Bucurescu, Phys. Rev. C 72, 044311 (2005).

[40] T. von Egidy and D. Bucurescu, Phys. Rev. C 80, 054310 (2009).

[41] S. Goriely et al., Phys. Rev. C 75, 064312 (2007).

[42] G. Audi et al., Nucl. Phys. A 729, 337 (2003).

[43] S. S. Dietrich and B. L. Berman, At. Data Nucl. Data Tables 38, 199 (1988).

[44] J. Kopecky, Handbook for calculations of nuclear reaction data, IAEA-TECDOC-1034, 97 (1998).

[45] C. Granja et al., Nucl. Phys. A 724, 14 (2003).

[46] A. Chyzh et al., Phys. Rev. C 84, 014306 (2011).

[47] B. Baramsai et al., Phys. Rev. C 87, 044609 (2013).

[48] J. Kroll et al., Phys. Scr. T 154, 014009 (2013).

[49] C. Schlegel et al., Phys. Lett. B 375, 21 (1996).

[50] S. F. Mughabghab, Atlas of Neutron Resonances, Elsevier, Amsterdam, (2006).

[51] D. A. Bohle et al., Phys. Lett. B 148, 260 (1984).

[52] P. G. Hansen et al., Nucl. Phys. A 518, 13 (1990).

[53] R. F. Casten et al., Phys. Rev. Lett. 58, 658 (1987).

[54] J. N. Ginocchio, Phys. Lett. B 265, 6 (1991).

[55] P. von Neumann-Cosel et al., Phys. Rev. Lett. 75, 4178 (1995).

[56] J. Kroll et al., in Proc. Int. Conf. on Nucl. Data for Sci. and Tech., New York, USA, Nuclear

Data Sheets, accepted (2014). 